\title{
Luminescence mechanism and energy transfer in cesium metavanadate $\mathrm{CsVO}_{3}$
}

\author{
A.V. Ishchenko ${ }^{a, *}$, K.V. Ivanovskikh ${ }^{a}$, I.A. Weinstein ${ }^{a}$, R.F. Samigullina ${ }^{b}$, V.V. Platonov ${ }^{c}$ \\ ${ }^{a}$ Ural Federal University, 19 Mira St., 620002, Ekaterinburg, Russia \\ ${ }^{\mathrm{b}}$ Institute of Solid State Chemistry, Ural Branch of the Russian Academy of Sciences, 91 Pervomayskaya St., 620990, Ekaterinburg, Russia \\ ${ }^{\mathrm{c}}$ Institute of Electrophysics, Ural Branch of the Russian Academy of Sciences, 106 Amundsena St., 620016, Ekaterinburg, Russia
}

\section{A R T I C L E I N F O}

\section{Keywords:}

Cesium vanadate

Metavanadate

Self-activated phosphor

Vibronic structure

Electron-phonon coupling

\begin{abstract}
A B S T R A C T
Photoluminescence properties and relaxation dynamics of electronic excitations in cesium vanadate $\mathrm{CsVO}_{3} \mathrm{have}$ been studied upon pulse laser excitation in the wide temperature range of $6.5-300 \mathrm{~K}$. A vibronic structure observed in low-temperature PL spectra is considered and interpreted. Peculiarities of luminescence relaxation dynamics and emission spectra of $\mathrm{CsVO}_{3}$ along with the appearance of the vibronic structure are explained in terms of strong electron-phonon coupling of excited electronic states and centrosymmetric vibrational modes in $\left[\mathrm{VO}_{4}\right]^{3-}$ center. A new approach in an interpretation of luminescence processes in vanadates is demonstrated.
\end{abstract}

\section{Introduction}

Vanadates consisting of $\left[\mathrm{VO}_{4}\right]^{3-}$ tetrahedron groups are well known as self-activated luminescent materials characterized by broad emission bands, high luminescence efficiency, good chemical stabilities, and radiation resistance. Among them, cesium vanadate $\mathrm{CsVO}_{3}$ is a promising multifunctional compound that can be synthesized through various relatively easy applicable synthesis techniques. Particularly, cesium vanadate attracts considerable attention due to the presence of unique properties related to the strong self-activated broadband luminescence that may be applied for conversion of UV and other types of ionizing radiation into the yellow-green emission (Matsuura et al., 2017; Nakajima et al., 2009, 2015). In addition, the compounds of such type show promise as phosphors for X-ray imaging, UV dosimetry, and white LED with blue or near UV-excitation (Nakajima et al., 2014; Slobodin et al., 2011, 2014).

According to the prevailing theoretical model the intrinsic luminescence in vanadate $\left(\mathrm{V}^{5+}\right)$ compounds, particular in cesium vanadate $\mathrm{CsVO}_{3}$, is due to the electronic transitions from the ${ }^{3} \mathrm{~T}_{1}$ and ${ }^{3} \mathrm{~T}_{2}$ triplet excited states to the ${ }^{1} \mathrm{~A}_{1}$ ground state (in terms of molecular orbital theory) in $\left[\mathrm{VO}_{4}\right]^{3-}$ groups (Ronde and Blasse, 1978). However, the many experimental data accumulated over the last decades did not show a good agreement with such simple theoretical model (Blasse, 1997; Ishchenko et al., 2016; Zubkov et al., 2009). So, the nature of relaxation process occurred from the excited states of $\left[\mathrm{VO}_{4}\right]^{3-}$ groups has not been fully understood so far. In this report, we present the preliminary results on time-resolved photoluminescence study aimed to get insight into the emission mechanism and energy transfer in $\mathrm{CsVO}_{3}$. Samples for this study have been synthesized through the solid state reaction techniques followed by modification through laser ablation. The laser ablation technique has been used to produce $\mathrm{CsVO}_{3}$ powder with particles that measure less than $1 \mu \mathrm{m}$ and to test luminescence properties of $\mathrm{CsVO}_{3}$ powder for dependence on the particle size.

\section{Samples and equipment}

The cesium metavanadate $\mathrm{CsVO}_{3}$ was synthesized through the solidstate reaction techniques by melting the stoichiometric mixture of $\mathrm{Cs}_{2} \mathrm{CO}_{3}$ and $\mathrm{V}_{2} \mathrm{O}_{5}$ raw materials at $670{ }^{\circ} \mathrm{C}$ for $2 \mathrm{~h}$. After cooling, the sample was ground thoroughly and kept at $560^{\circ} \mathrm{C}$ for $10 \mathrm{~h}$ to remove the stresses in the calcined material. As a result, microcrystalline $\mathrm{CsVO}_{3}$ powder with grain size ranged from about 10 to $80 \mu \mathrm{m}$ was obtained. Next, we applied a laser ablation technique (Osipov et al., 2006) in order to produce submicrocrystalline powders with dominating grain size ranged from 0.1 to $0.3 \mu \mathrm{m}$ and presence of large particles with average size of few microns. The structure type and phase purity of the synthesized samples were characterized with conventional powder Xray diffraction method. No traces of impurity phases were found in the synthesized samples. Morphology of the samples produced after the solid-state reaction and after the laser ablation was examined using scanning electron microscopy (SEM) (Fig. 1). Particles of the powder obtained after solid-state synthesis demonstrate complex irregular

\footnotetext{
* Corresponding author.

E-mail address: a-v-i@mail.ru (A.V. Ishchenko).
} 

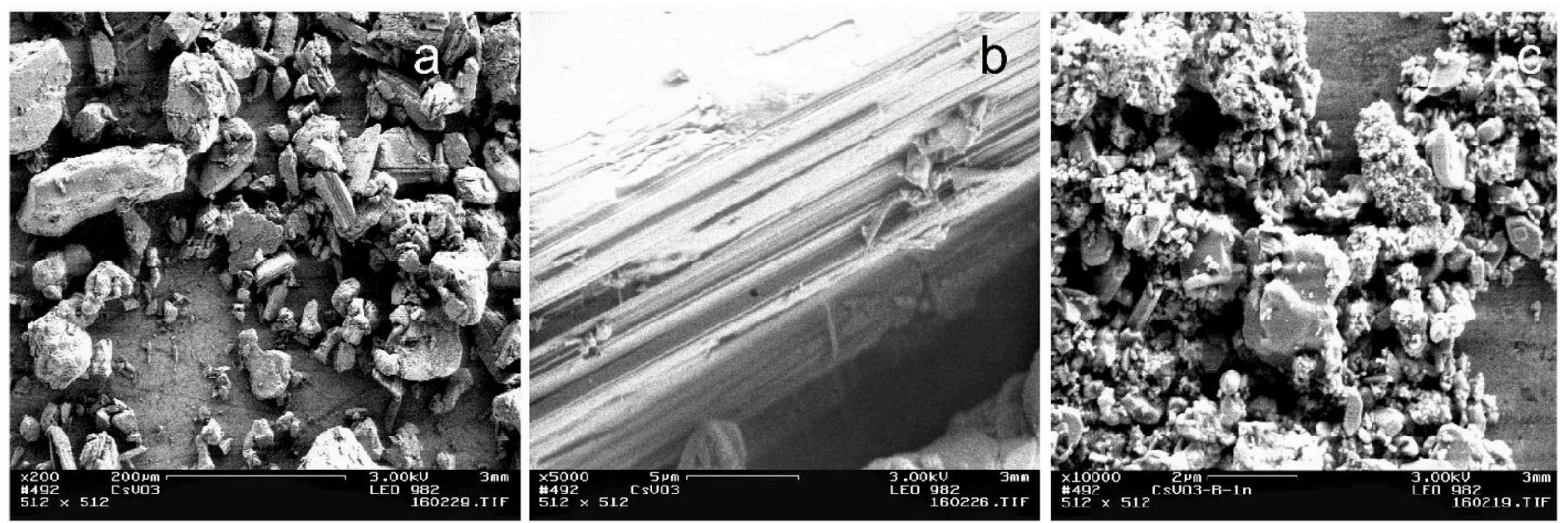

Fig. 1. The SEM images of $\mathrm{CsVO}_{3}$ samples synthesized by solid-state reaction (a, b) and modified by laser ablation (c).

shapes with a layered structure (Fig. 1a and b). The submicrocrystalline powder obtained after densification of $\mathrm{CsVO}_{3}$ and laser ablation technique is found to dominate by rounded-shaped particles which tend to aggregate (Fig. 1,c). Note particles sized from 0.5 to $2 \mu \mathrm{m}$ are mostly fragments of initial larger particles which could not be well destroyed by the laser.

The photoluminescence (PL) and photoluminescence excitation (PLE) spectra were measured at $300 \mathrm{~K}$ using a PerkinElmer LS-55 luminescence spectrometer equipped with a pulse Xe arc lamp as an excitation source. The PLE spectra were corrected using Lumogen F Red 300 luminescence dye (BASF). PL spectra and decay curves measurements at 6-300 K were performed using a DTL-380QT pulse $266 \mathrm{~nm}$ (fourth harmonic) YLF:Nd laser (pulse duration $<10 \mathrm{~ns}$ at $266 \mathrm{~nm}$ ) (Laser-Export Co. Ltd., Russia) and Janis CCS-100-204N vacuum cryostat at NANOTECH Centre, Ural Federal University.

The low-temperature PL spectra were recorded using an Andor Shamrock 303i monochromator equipped with an Andor Newton 970 EMCCD camera. The low-temperature decay curves were recorded using a Hamamatsu R2693 PMT and a LeCroy HDO 6104MS oscilloscope operated in TCSPC mode.

Decay curves upon pulse excitation at 230 and $370 \mathrm{~nm}$ at $300 \mathrm{~K}$ were recorded at Utrecht University (the Netherlands) using an Ekspla NT 342B optical parametric oscillator laser and a Jobin Yvon Triax 550 single emission monochromator equipped with a 1200 lines $/ \mathrm{mm}$ grating blazed at $400 \mathrm{~nm}$, a Hamamatsu R928 photomultiplier tube and a EG\&G Ortec Turbo-MCS multichannel scaler.

Experimental results were approximated using a chi-square approach. The luminescence spectra were corrected for $\lambda^{2}$ and $E^{3}$ according to (De Jong et al., 2015).

\section{Results and discussion}

\subsection{Photoluminescence}

The PL spectrum of cesium vanadate $\mathrm{CsVO}_{3}$ (Fig. 2-a) recorded at $300 \mathrm{~K}$ upon excitation with a pulse Xe lamp at $3.35 \mathrm{eV}(370 \mathrm{~nm})$ demonstrates broad emission band with maximum at $2.38 \mathrm{eV}(520 \mathrm{~nm})$ and FWHM of about $0.65 \mathrm{eV}$ that is typical for other vanadates of different structure and composition as documented elsewhere (Blasse, 1997; Ishchenko et al., 2016; Nakajima et al., 2009; Nakajima et al., 2014; Ronde and Blasse, 1978). The shape of the PL spectrum does not depend on excitation energy within the range of 3.0-6.0 eV. The PLE spectrum (Fig. 2-a) recorded monitoring emission at $2.38 \mathrm{eV}$ is represented by an onset just above $3 \mathrm{eV}$ followed by a superposition of complex excitation bands. It is important to point out that the three excitation peaks centered near 3.37, 3.57, $3.77 \mathrm{eV}$ are separated from each other by $0.2 \mathrm{eV}$ (Fig. 2-a).

We note that we found no difference in luminescence spectroscopic properties of microcrystalline and submicrocrystalline $\mathrm{CsVO}_{3}$ powders in contrast to $\alpha-\mathrm{Zn}_{2} \mathrm{~V}_{2} \mathrm{O}_{7}$ pyrovanadate; while latter demonstrated noticeable variation of luminescence properties as documented by some of us (Ishchenko et al., 2016). Therefore, we omit using 'microcrystalline' or 'submicrocrystalline' terms below when describing experimental results below.

Fig. 2-b represents a series of temperature dependent emission spectra recorded upon excitation with pulse laser at $4.66 \mathrm{eV}(266 \mathrm{~nm})$. When temperature decreases from $300 \mathrm{~K}$ to $6.5 \mathrm{~K}$ the emission band demonstrates gradual redshift of its barycenter for about $0.05 \mathrm{eV}$ and narrowing along with the appearance of the well distinguished vibronic structure. The latter is discussed in Section 3.3.

It is widely accepted that the self-activated luminescence of vanadates arises from spin-forbidden triplet-singlet transitions between ${ }^{3} \mathrm{~T}_{2}$ and ${ }^{3} \mathrm{~T}_{1}$ lower excited states and ${ }^{1} \mathrm{~A}_{1}$ ground state after excitation into the high energy states ${ }^{1} \mathrm{~T}_{2}$ and ${ }^{1} \mathrm{~T}_{1}$. The ${ }^{1} \mathrm{~A}_{1}$ ground state corresponds to the highest completely filled non-bonding (oxygen-localized $2 \mathrm{p}$ states) $\mathrm{t}_{1}$ molecular orbital. The excited states ${ }^{1} \mathrm{~T}_{2}$ and ${ }^{1} \mathrm{~T}_{1}$ belong to the first excited $t_{1}{ }^{5} 2$ e configuration, where the antibonding $2 \mathrm{e}$ level has mainly $3 \mathrm{~d}$ vanadium character. The ground and excited states are notated in terms of molecular orbitals theory for $\left[\mathrm{VO}_{4}\right]^{3-}$ anion groups (Ronde and Blasse, 1978). However, it should be noted that aforementioned model and related calculations of electronic structure are made for vanadates and other oxides with transition metals (tungstates, niobates, etc.) while suggesting non-periodical molecular structure with isolated ions (Ronde and Blasse, 1978) and excluding any influence of cationic sublattice. Such a model well describes only optical absorption properties of $\mathrm{V}_{2} \mathrm{O}_{5}$ molecule dissociated in aqueous solution (Muller et al., 1969; Ronde and Blasse, 1978). In this connection, the luminescence properties of condensed vanadates cannot be fully described in terms of molecular orbitals theory (Blasse, 1997). Nevertheless, considering results of computational quantum mechanical modeling of vanadate band structure (Qiao et al., 2016) and the abovementioned model it can be concluded that the PLE spectrum of cesium vanadate (Fig. 2, a) arises from charge transfer (CT) transitions $\mathrm{O}(2 \mathrm{p}) \rightarrow \mathrm{V}(3 \mathrm{~d})$. The specific shape of the PLE spectrum is defined by features of vanadium 3d-orbital crystal field splitting. The PL spectrum of $\mathrm{CsVO}_{3}$ can be assigned to relaxation of disturbed anion groups to the ground state $\left(\left[\mathrm{VO}_{4}\right]^{3-}\right.$ )$^{*} \rightarrow\left[\mathrm{VO}_{4}\right]^{3-}$. The complete structure of excited states is difficult to determine at the present time.

\subsection{Relaxation dynamics}

Fig. 3-a represents luminescence decay curves recorded upon pulse laser excitation at $266 \mathrm{~nm}$ while monitoring luminescence at $2.88 \mathrm{eV}$ $(430 \mathrm{~nm})$ at the different temperatures. We note that identical decay curves are obtained when emission is taken at $1.91 \mathrm{eV}(650 \mathrm{~nm})$ (not shown for brevity). 

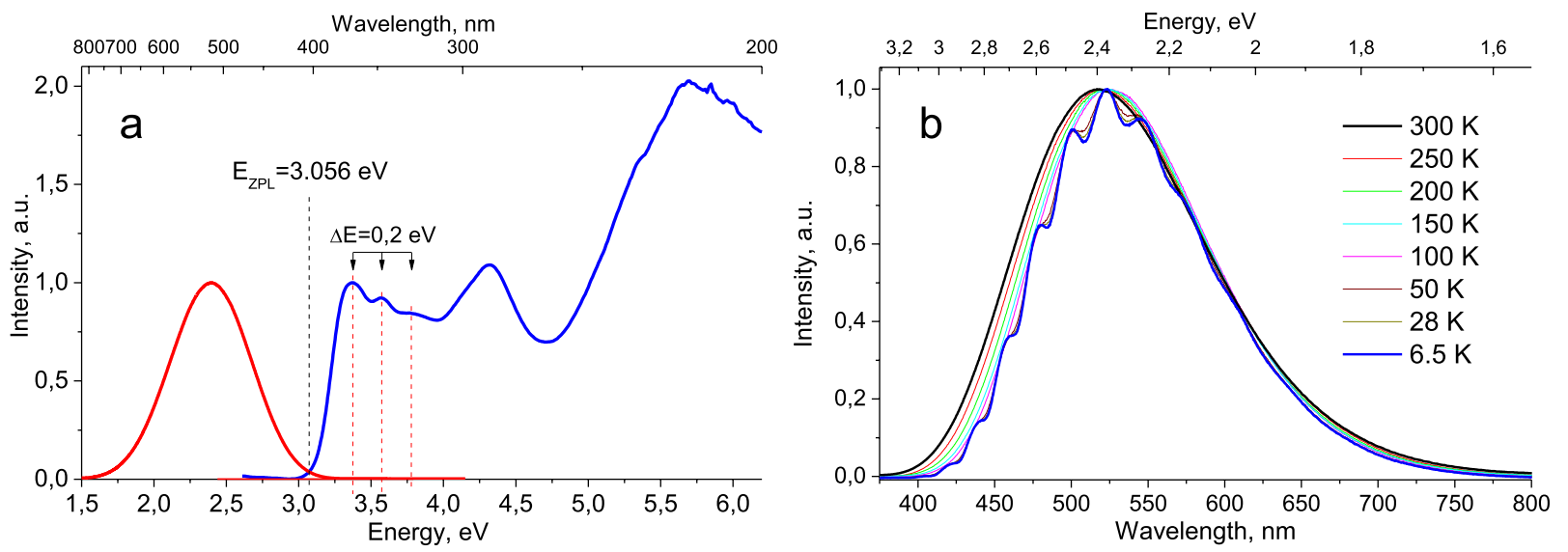

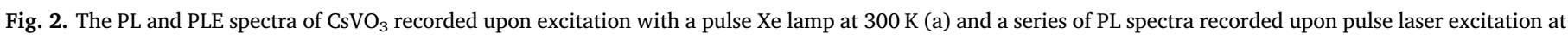
$266 \mathrm{~nm}$ in the temperature range of $6.5-300 \mathrm{~K}$ (b).

The obtained decay curves could not be satisfactory fitted with a single or a double exponential decay function. While successful fitting could be achieved by involving the third exponential decay components we point out that an approximation of decay curves with three and more exponential decay components is quite often provides just a mathematical result rather than describes real physical processes. Therefore, in order to evaluate temperature dependence of luminescence relaxation dynamics the average emission lifetime was calculated using the equation:

$\tau_{\text {avg }}=\frac{A_{1} \tau_{1}^{2}+A_{2} \tau_{2}^{2}+A_{3} \tau_{3}^{2}}{\left(A_{1} \tau_{1}+A_{2} \tau_{2}+A_{3} \tau_{3}\right)}$

where $\tau_{\mathrm{i}}$ is a lifetime of an exponential decay component with an amplitude of $A_{\mathrm{i}}$. The calculated decay parameters are shown in Table 1 .

The temperature dependence of average emission lifetime $\left(\tau_{\mathrm{av}}\right)$ of $\mathrm{CsVO}_{3}$ (see Table 1 and Fig. 3-b) can be fitted by the Mott law (Shalapska et al., 2010)

$$
\tau=\frac{\tau_{0}}{1+\tau_{0} \Gamma \exp \left(-\frac{E_{a}}{k T}\right)},
$$

where $E_{\mathrm{a}}$ is activation energy for the thermal quenching, $\tau_{0}$ is radiative decay time, $\Gamma$ is attempt rate and $k$ is Boltzmann constant. A satisfactory fitting can be achieved using the following parameters: $\tau_{0}=1.21 \mathrm{~ms}$, $\Gamma=4.69 \cdot 10^{5} \mathrm{~s}^{-1}$ and $E_{\mathrm{a}}=40 \mathrm{meV}$. We suppose that a relatively low value of the activation energy $E_{\mathrm{a}}$ indicates the existence of exciton-like charge transfer excitations in cesium vanadate. To get this assumption well supported further theoretical and experimental studies are required.

PL decay curves recorded upon excitation with OPO laser at $3.35 \mathrm{eV}$ $(370 \mathrm{~nm})$ clearly and $5.39 \mathrm{eV}(230 \mathrm{~nm})$ demonstrate that relaxation dynamics has a different character when excited just above the onset of the excitation spectrum (Fig. 4-a) and far above that (Fig. 4-b). In particular, the decay kinetics recorded upon excitation at $5.39 \mathrm{eV}$ demonstrate the appearance of faster decay component with the estimated lifetime of from 1 to $5 \mu$ s and slower decay component with the estimated lifetime of about $120 \mu \mathrm{s}$. The latter tends to become more pronounced for the high-energy side of the $\mathrm{CsVO}_{3}$ emission band.

The observed phenomena suggest that we witness the presence of complex excitation and relaxation process related to $\left[\mathrm{VO}_{4}\right]^{3-}$ group. We suppose that apart from the formation of conventional CT state, excitation of $\left[\mathrm{VO}_{4}\right]^{3-}$ groups with high-energy photons may lead to ionization $\left[\mathrm{VO}_{4}\right]^{3-}$ groups and creation of metastable exciton like ([ $\left.\mathrm{VO}_{4}\right]^{2-}$ $+\mathrm{e}^{-}$)-centers. From the one hand, the presence of competitive process results in quenching of the CT emission band; from the other hand, retrapping of electrons released after non-radiative relaxation of $\left(\left[\mathrm{VO}_{4}\right]^{2-}+\mathrm{e}^{-}\right)$exciton-like states results in the appearance of the delayed luminescence (that is observed upon excitation at $5.39 \mathrm{eV}$, Fig. 4b). We note that an important condition for having such exciton-like states realized is the presence of strong exciton-phonon interaction. Below we demonstrate that this is well realized in $\mathrm{CsVO}_{3}$. To further investigate the observed phenomena and to test the assumption about their origin, further experiments involving temperature and excitation energy dependent measurements of decay kinetics are required.
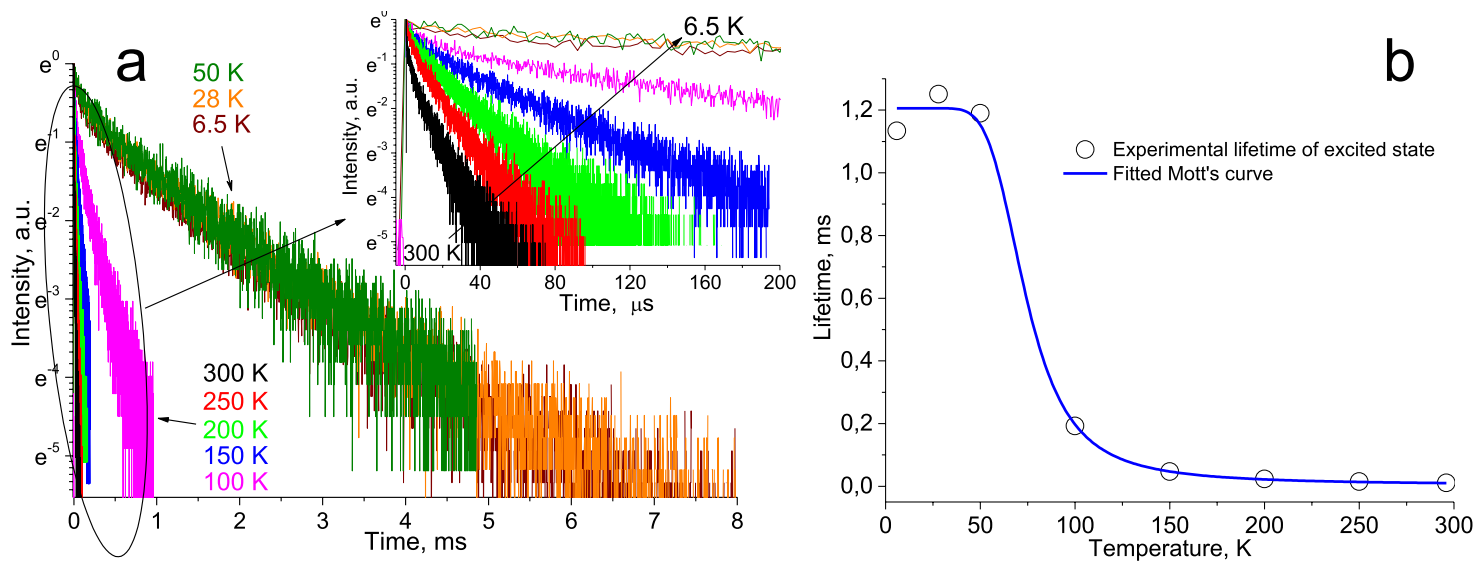

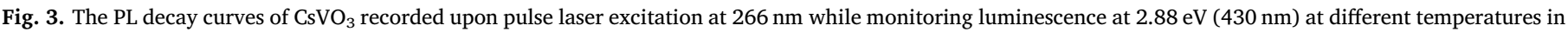
the range of $6.5-300 \mathrm{~K}$ (a) and temperature dependence of average lifetime of $\mathrm{CsVO}_{3} \mathrm{PL}$ along with fitting curve obtained using the Mott's relation (2). 
Table 1

The decay parameters of $\mathrm{CsVO}_{3}$ luminescence upon pulse laser excitation at $266 \mathrm{~nm}$ in the temperature range of $6.5-300 \mathrm{~K}$.

\begin{tabular}{llllllll}
\hline $\mathrm{T}, \mathrm{K}$ & $\tau_{1}, \mu \mathrm{s}$ & $\mathrm{A}_{1}$, a.u. & $\tau_{2}, \mu \mathrm{s}$ & $\mathrm{A}_{2}$, a.u. & $\tau_{3}, \mathrm{~ms}$ & $\mathrm{~A}_{3}$, a.u. & $\tau_{\mathrm{av}}, \mu \mathrm{s}$ \\
\hline 300 & 0.45 & 0.36 & 2.69 & 0.26 & 0.013 & 0.39 & 10.8 \\
250 & 1.11 & 0.26 & 4.89 & 0.25 & 0.175 & 0.48 & 15.2 \\
200 & 0.77 & 0.26 & 5.86 & 0.24 & 0.026 & 0.50 & 23.4 \\
150 & 2.67 & 0.16 & 15.4 & 0.28 & 0.051 & 0.56 & 47.3 \\
100 & 8.66 & 0.32 & 48.4 & 0.47 & 0.220 & 0.20 & 192 \\
50 & 45.5 & 0.53 & 312 & 0.26 & 1.31 & 0.21 & 1190 \\
28 & 36.6 & 0.32 & 339 & 0.45 & 1.42 & 0.23 & 1250 \\
6.5 & 50.8 & 0.49 & 274 & 0.28 & 1.26 & 0.23 & 1130 \\
\hline
\end{tabular}

\subsection{Analysis of the vibronic structure}

According to the molecular orbital theory, the luminescence spectra of cesium vanadate should have two emission bands assigned to ${ }^{3} \mathrm{~T}_{2} \rightarrow{ }^{1} \mathrm{~A}_{1}$ and ${ }^{3} \mathrm{~T}_{1} \rightarrow{ }^{1} \mathrm{~A}_{1}$ transitions. We note, however, that the PL spectra recorded at $300 \mathrm{~K}$ can only be fitted with a single Gaussian shaped band (Fig. 5-a). The same is true for the PL spectrum taken at low temperatures (below $100 \mathrm{~K}$ ) which also demonstrate the presence of the vibronic structure.

Usually, to describe vibronic bands in luminescence spectra of impurity centers an equation for transition moment squared is used (De Jong et al., 2015; Sapozhnikov, 1976):

$W(E)=W_{0} \sum_{n} F_{n}^{0} f(E, n, \sigma)$,

where $\mathrm{W}_{0}$ is the square of the transition moment of the electronic transition. The summation describes the distribution of the intensity over the individual vibronic lines. The shape of the distribution is defined by the type of $F_{n}^{0}$ function. The $f$ functions describe the shape of each individual line (vibronic replica), centered on $E \cdot n$ with a width $\sigma$, where $n$ is the number of vibronic replicas (vibronic quantum number). $F_{n}^{0}$ function expressed for the impurity center is represented by a Poisson distribution and is defined as

$F_{n}^{0}=\left|\int \psi_{0}^{*} \psi_{n} \mathrm{~d} Q\right|^{2}=\frac{e^{-S} S^{n}}{n !}$, where $\psi_{\mathrm{i}}$ are wave functions of electronic ground and excited states, $S$ is Huang-Rhys factor and $n$ is the vibrational quantum number.

In contrast to phosphors with impurity centers vanadates are selfactivated phosphors as luminescence centers are embedded into the regular lattice. Unfortunately, for such phosphors the analytical expression for $F_{n}^{0}$ is not clear. The attempts of fitting the experimental data using equations (3) and (4) with the Gaussian shape of individual vibronic replica $(f(E, n, \sigma)$ function) failed. Therefore, we tried to describe the experimental data by equation (3) with the free shape of $F_{n}^{0}$ function using the following expression

$W(E)=W_{0} \sum_{n} F_{n} \cdot e^{-\frac{\left(E-\bar{E}_{n}\right)^{2}}{2 \Delta w^{2}}}$,

where $F_{n}$ are variable values which represent intensity for the individual vibronic replica, $\Delta w$ is spectral width of Gaussian-shaped individual vibronic line, $\bar{E}_{n}=E_{Z P L}-\hbar \omega(n+1 / 2)$ is average (effective) center of individual vibronic lines, while $E_{Z P L}$ is energy of zero-phonon line and $\hbar \omega$ is phonon energy. Other parameters have been described above. The results of such approximation are presented in Fig. 5-b and Fig. 6. The obtained values are $E_{Z P L}=3.074 \mathrm{eV}, \hbar \omega=111 \mathrm{meV}$ $\left(893 \mathrm{~cm}^{-1}\right)$. The values of $F_{n}$ and $\Delta w$ are shown in Fig. 6 as dependences on $n$. It is clearly seen that distribution of $F_{n}$ function has a Gaussian shape that is also confirmed by the fitting procedure (Fig. 6). So, considering these results, we can propose an expression for the $F_{n}^{0}$ function in equation (3) that describes the vibronic structure in luminescence spectra of cesium vanadate:

$F_{n}^{0}=\left|\int \psi_{0}^{*} \psi_{n} \mathrm{~d} Q\right|^{2}=e^{-\frac{(n-S)^{2}}{2 \Delta s^{2}}}$

where $\Delta s$ characterizes the range of Huang-Rhys parameter distribution. Finally, the approximation gives rise the results of $S=6.48$ and $\Delta s=2.268$. The high value of $S$ indicates the strong electron-phonon coupling in cesium vanadate. The physical meaning of $\Delta s$ parameter would require further refining and clarification.

As can be seen from Fig. 6- $b$ the spectral width of individual vibronic lines in PL spectra tends to rise when the vibrational quantum number increases. To understand this dependence we should consider the following peculiarities. According to (Kourouklis et al., 1991) there are four Raman frequencies observed at 657, 914, 938 and $943 \mathrm{~cm}^{-1}$,
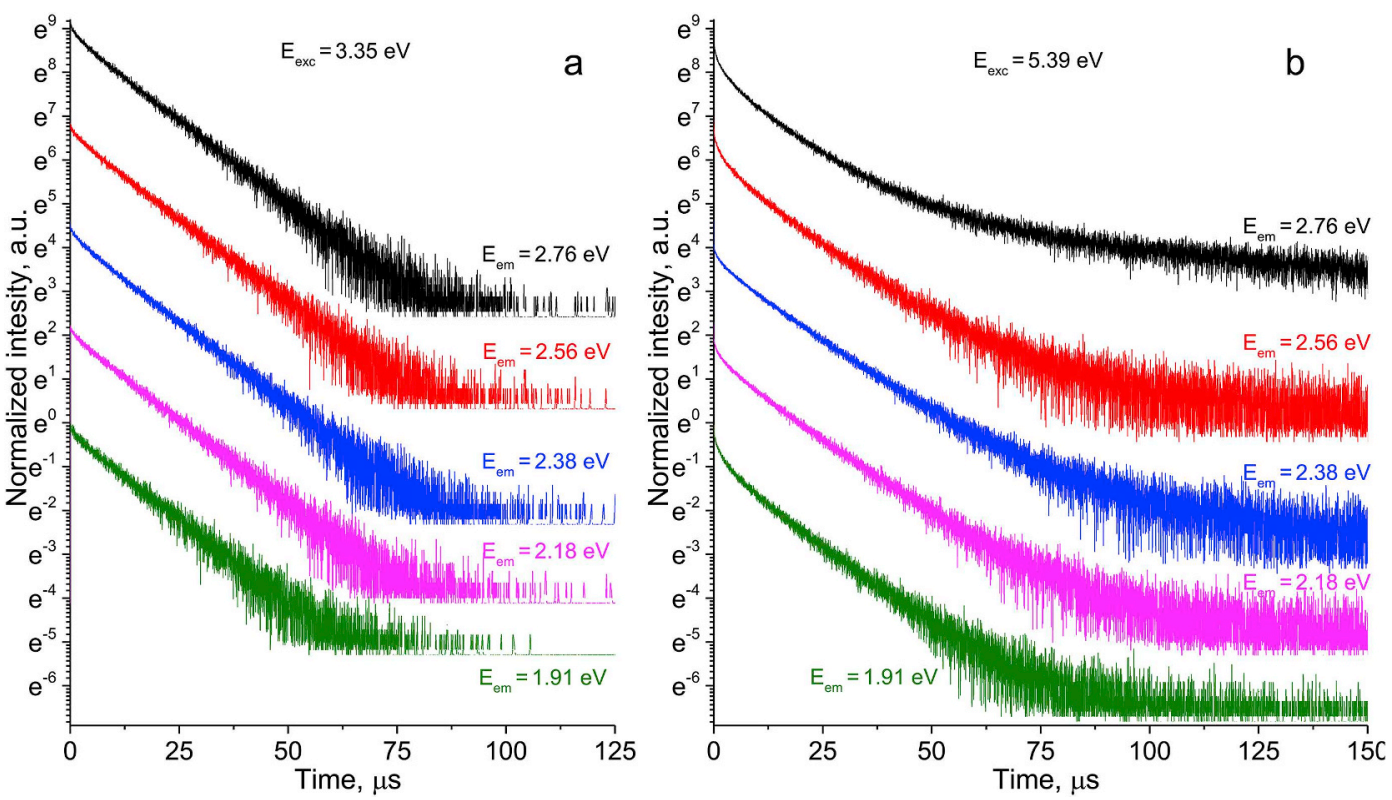

Fig. 4. The PL decay curves of $\mathrm{CsVO}_{3}$ luminescence monitored at 1.91, 2.18, 2.38, 2.56 and $2.76 \mathrm{eV}(650,570,520,485$ and $450 \mathrm{~nm}$, respectively) upon pulse OPO laser excitation $3.35 \mathrm{eV}(370 \mathrm{~nm})$ (a) and $5.39 \mathrm{eV}(230 \mathrm{~nm})(\mathrm{b})$ at $\mathrm{T}=300 \mathrm{~K}$. 

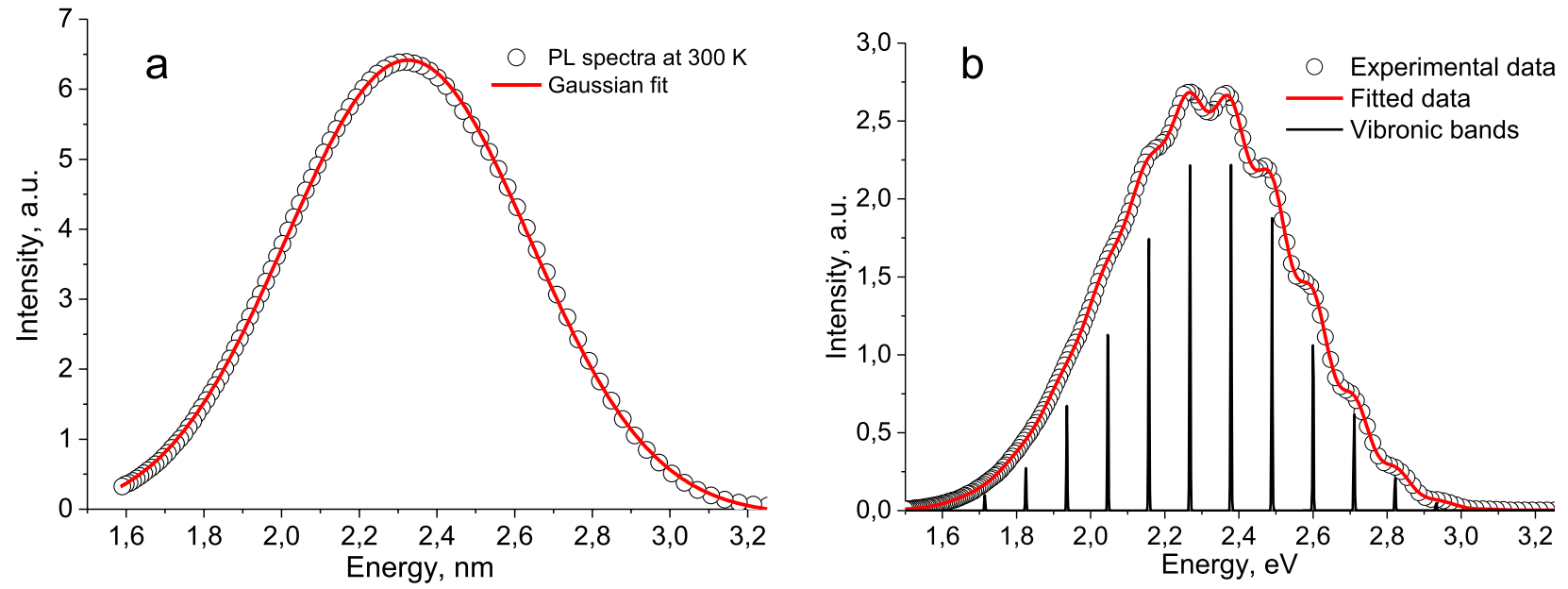

Fig. 5. PL experimental spectra recorded at 300 (a) and $6.5 \mathrm{~K}$ (b) with fitted theoretical spectra.
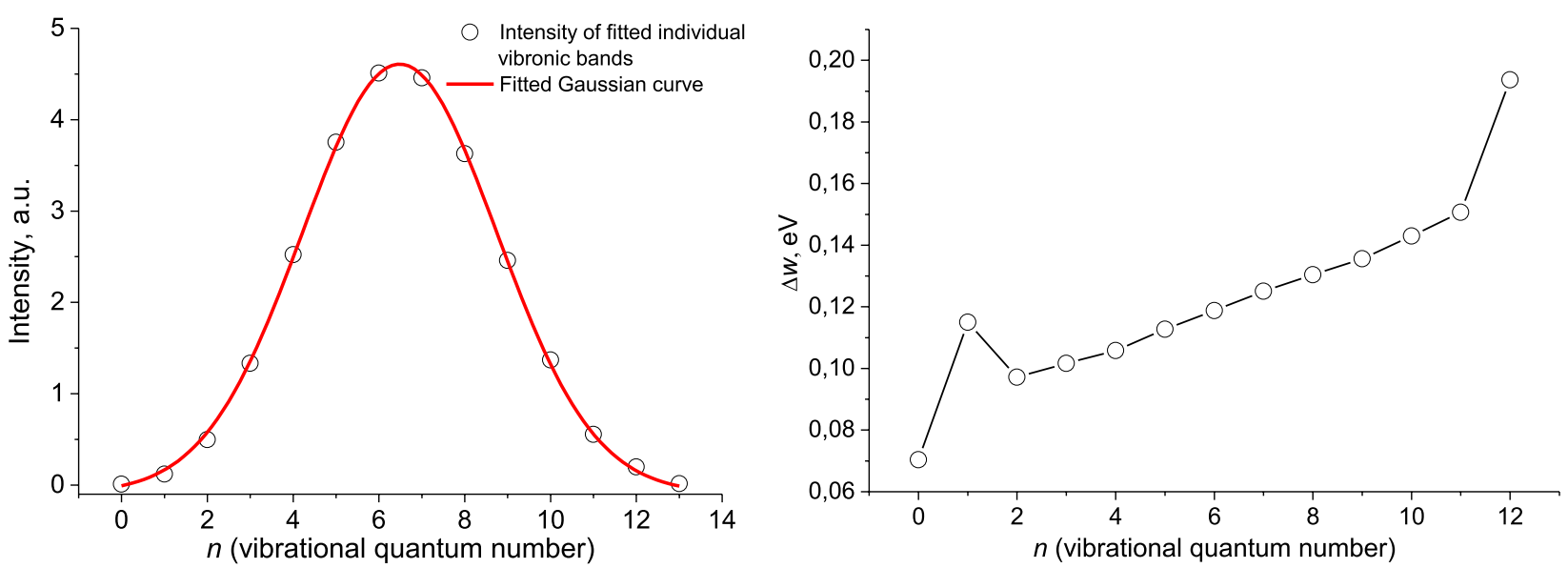

Fig. 6. Results of the fitting procedure for intensity (a) and spectral width (b) of individual vibronic bands.

which are assigned to centrosymmetric $\mathrm{O}-\mathrm{V}-\mathrm{O}$ and $\mathrm{V}=\mathrm{O}$ vibrational modes in $\left[\mathrm{VO}_{4}\right]^{3-}$ center. In fact, the modeled vibrational series (Fig. 5b) represent a superposition of four aforementioned vibrational modes in terms of electron-phonon coupling with the single vibrational mode. Thus, the obtained value of $\hbar \omega=111 \mathrm{meV}\left(893 \mathrm{~cm}^{-1}\right)$ is averaged or effective phonon energy of the four abovementioned Raman frequencies taken according to their intensity in the Raman spectrum. For the low values of $n$, the shift between vibrational modes with Raman frequencies of $657,914,938$ and $943 \mathrm{~cm}^{-1}$ relative to the ZPL is still insignificant. In this case, no significant broadening of the averaged individual vibronic line is observed and as a result the vibronic structure is well pronounced in PL spectra (Fig. 5-b). As the vibrational quantum number increases the shift of Raman frequencies raises that in turn leads to broadening of the averaged individual vibronic line as well (Fig. 6-b). Eventually, the vibronic structure in PL spectra becomes gradually diminishes (Fig. 5-b).

Finally, it should be noted that at low temperatures the individual vibronic lines are typically narrow ( $\sigma$ is small) that provides observation of distinct peaks, while at room temperature $\sigma$ is larger that makes the peaks overlap into a broadband. So, as temperature increases from 6 to $300 \mathrm{~K}$, the vibronic structure in PL spectra of $\mathrm{CsVO}_{3}$ becomes less visible and broadband spectrum with smoothed vibronic bands is observed. We suppose that the above-described model can be expanded and tested for wider vanadate family.

\section{Conclusion}

In present work, we have demonstrated a new approach for interpretation of luminescence processes in vanadates. The appearance of vibronic lines in low-temperature PL spectra of cesium vanadate $\mathrm{CsVO}_{3}$ is considered and interpreted. The intensity of individual vibronic lines is experimentally shown to obey a Gaussian distribution. The vibronic replicas in PL spectra of $\mathrm{CsVO}_{3}$ are assigned to strong electron-phonon coupling of the excited electronic states and centrosymmetric vibrational modes in $\left[\mathrm{VO}_{4}\right]^{3-}$ center. The observed difference in luminescence relaxation dynamics of CT states in $\mathrm{CsVO}_{3}$ is explained in terms of proposed strong electron-phonon coupling model.

\section{Acknowledgement}

The work was supported by Act 211 Government of the Russian Federation, contract № 02.A03.21.0006. I.A.W. thanks for financial support Minobrnauki initiative research project № 16.5186.2017/8.9. Authors thank Dr. Mathijs de Jong (Utrecht University) for his kind assistance in laser experiments and Prof. Boris V. Shulgin for fruitful discussions of this work.

\section{Appendix A. Supplementary data}

Supplementary data to this article can be found online at https:// doi.org/10.1016/j.radmeas.2019.03.003. 


\section{References}

Blasse, G., 1997. Classical phosphors: a Pandora's box. J. Lumin. 72-74, 129-134. De Jong, M., Seijo, L., Meijerink, A., Rabouw, F.T., 2015. Resolving the ambiguity in the relation between Stokes shift and Huang-Rhys parameter. Phys. Chem. Chem. Phys. $17,16959-16969$.

Ishchenko, A.V., Samigullina, R.F., Krasnenko, T.I., Onufrieva, T.A., Shulgin, B.V., 2016. Influence of grain size on luminescence properties of micro- and nanopowder $\mathrm{Zn}_{2} \mathrm{~V}_{2} \mathrm{O}_{7}$ vanadate. Radiat. Meas. 90, 33-37.

Kourouklis, G.A., Jayaraman, A., Espinosa, G.P., Cooper, A.S., 1991. High-pressure Raman study of $\mathrm{CsVO}_{3}$ and pressure-induced phase transitions. J. Raman Spectrosc. $22,57-60$.

Matsuura, T., Miyazaki, H., Ota, T., 2017. Low-temperature synthesis of white-lightemitting $\mathrm{CsVO}_{3}$ nanoparticles by an aqueous solution route. J. Ceram. Soc. Jpn. 125, 657-659.

Muller, A., Diemann, E., Ranade, A.C., 1969. The electronic spectra of $\mathrm{VO}_{4}{ }^{3-}, \mathrm{VS}_{4}{ }^{3-}$ and $\mathrm{VSe}_{4}{ }^{3-}$. Chem. Phys. Lett. 3, 467-468.

Nakajima, T., Isobe, M., Tsuchiya, T., Ueda, Y., Kumagai, T., 2009. A revisit of photoluminescence property for vanadate oxides $\mathrm{AVO}_{3}$ (A: K, Rb and $\mathrm{Cs}$ ) and $\mathrm{M}_{3} \mathrm{~V}_{2} \mathrm{O}_{8}(\mathrm{M}$ : $\mathrm{Mg}$ and Zn). J. Lumin. 129, 1598-1601.

Nakajima, T., Isobe, M., Uzawa, Y., Tsuchiya, T., 2015. Rare earth-free high color rendering white light-emitting diodes using $\mathrm{CsVO}_{3}$ with highest quantum efficiency for vanadate phosphors. J. Mater. Chem. C. 3, 10748-10754.

Nakajima, T., Shinoda, K., Tsuchiya, T., 2014. Single-LED solar simulator for amorphous
Si and dye-sensitized solar cells. RSC Adv. 4, 19165-19171.

Osipov, V.V., Kotov, YuA., Ivanov, M.G., Samatov, O.M., Lisenkov, V.V., Platonov, V.V., Murzakaev, A.M., Medvedev, A.I., Azarkevich, E.I., 2006. Laser synthesis of nanopowders. Laser Phys. 16, 116-125.

Qiao, X., Li, Y., Wan, Y., Huang, Y., Cheng, H., Seo, H.J., 2016. Preparation, characterization and high quantum efficiency of yellow-emitting CsVO3 nanofibers. J. Alloy. Comp. 656, 843-848.

Ronde, H., Blasse, G., 1978. The nature of the electronic transitions of the vanadate group. J. Inorg. Nucl. Chem. 40, 215-219.

Sapozhnikov, M.N., 1976. Zero-phonon transitions in the optical spectra of impurity molecular crystals. Phys. Status Solidi 76, 11-51.

Shalapska, T., Stryganyuk, G., Gektin, A., Demchenko, P., Voloshinovskii, A., Dorenbos, P., 2010. Crystal structure and luminescence properties of $\mathrm{LiYP}_{4} \mathrm{O}_{12}: \mathrm{Ce}^{3+}$ phosphor. J. Phys. Condens. Matter 22, 485503.

Slobodin, B.V., Ishchenko, A.V., Samigullina, R.F., Teslenko, O.S., Shul'Gin, B.V., Zhurakovskii, D.Yu, 2011. Thermochemical and luminescent properties of $\mathrm{RbVO}_{3}$ $\mathrm{CsVO}_{3}$, and $\mathrm{Rb}_{0.5} \mathrm{Cs}_{0.5} \mathrm{VO}_{3}$. Inorg. Mater. 47, 1126-1131.

Slobodin, B.V., Ishchenko, A.V., Samigullina, R.F., Yagodin, V.V., Shul'Gin, B.V., 2014 Preparation and luminescent properties of rubidium and cesium vanadates. Inorg. Mater. 50, 179-183.

Zubkov, V.G., Tyutyunnik, A.P., Tarakina, N.V., Berger, I.F., Surat, L.L., Slobodin, B.V., Svensson, G., Forslund, B., Shulgin, B.V., Pustovarov, V.A., Ishchenko, A.V., Cherepanov, A.N., 2009. Synthesis, crystal structure and luminescent properties of pyrovanadates $\mathrm{A}_{2} \mathrm{CaV}_{2} \mathrm{O}_{7}(\mathrm{~A}=\mathrm{Rb}, \mathrm{Cs})$. Solid State Sci. 11, 726-732. 